\title{
Infliximab Efficacy May Be Linked to Full TNF- $\alpha$ Blockade in Peripheral Compartment-A Double Central-Peripheral Target-Mediated Drug Disposition (TMDD) Model
}

\author{
David Ternant ${ }^{1,2, *}$, Olivier Le Tilly ${ }^{1,2}$, Laurence Picon ${ }^{3}$, Driffa Moussata ${ }^{1,3}$, Christophe Passot ${ }^{4}$, \\ Theodora Bejan-Angoulvant ${ }^{1,2}$, Céline Desvignes ${ }^{1,2}{ }^{\circ}$, Denis Mulleman ${ }^{5,6} \oplus^{\circ}$, Philippe Goupille ${ }^{6}$ \\ and Gilles Paintaud ${ }^{1,2}$
}

check for

updates

Citation: Ternant, D.; Le Tilly, O.;

Picon, L.; Moussata, D.; Passot, C.;

Bejan-Angoulvant, T.; Desvignes, C.; Mulleman, D.; Goupille, P.; Paintaud, G. Infliximab Efficacy May Be Linked to Full TNF- $\alpha$ Blockade in Peripheral Compartment-A Double Central-Peripheral Target-Mediated Drug Disposition (TMDD) Model. Pharmaceutics 2021, 13, 1821. https:// doi.org/10.3390/pharmaceutics 13111821

Academic Editors: Zoubir Djerada and Xavier Delavenne

Received: 14 September 2021 Accepted: 19 October 2021

Published: 1 November 2021

Publisher's Note: MDPI stays neutral with regard to jurisdictional claims in published maps and institutional affiliations.

Copyright: (C) 2021 by the authors. Licensee MDPI, Basel, Switzerland. This article is an open access article distributed under the terms and conditions of the Creative Commons Attribution (CC BY) license (https:// creativecommons.org/licenses/by/ $4.0 /)$.
1 EA 4245 “Transplantation, Immunology, Inflammation”, Université de Tours, 37044 Tours, France; letilly@univ-tours.fr (O.L.T.); driffa.moussata@univ-tours.fr (D.M.); theodora.angoulvant@univ-tours.fr (T.B.-A.); celine.desvignes@univ-tours.fr (C.D.); gilles.paintaud@univ-tours.fr (G.P.)

2 Department of Clinical Pharmacology, CHRU de Tours, 37044 Tour, France

3 Department of Gastroenterology, CHRU de Tours, 37044 Tour, France; L.PICON@chu-tours.fr

4 Département de Biopathologie, Institut de Cancérologie de l'Ouest, 49055 Angers, France; Christophe.Passot@ico.unicancer.fr

5 EA 7501 “Groupe Innovation et Ciblage Cellulaire”, Université de Tours, 37044 Tour, France; denis.mulleman@univ-tours.fr

6 Department of Rheumatology, CHRU de Tours, 37044 Tour, France; philippe.goupille@univ-tours.fr

* Correspondence: david.ternant@univ-tours.fr

\begin{abstract}
Infliximab is an anti-TNF- $\alpha$ monoclonal antibody approved in chronic inflammatory bowel diseases (IBD). This study aimed at providing an in-depth description of infliximab targetmediated pharmacokinetics in 133 IBD patients treated with $5 \mathrm{mg} / \mathrm{kg}$ infliximab at weeks 0,2 , 14, and 22. A two-compartment model with double target-mediated drug disposition (TMDD) in both central and peripheral compartments was developed, using a rich database of 26 ankylosing spondylitis patients as a reference for linear elimination kinetics. Population approach and quasisteady-state (QSS) approximation were used. Concentration-time data were satisfactorily described using the double-TMDD model. Target-mediated parameters of central and peripheral compartments were respectively baseline TNF concentrations $\left(\mathrm{R}_{0} \mathrm{C}_{0}=3.3 \mathrm{nM}\right.$ and $\left.\mathrm{R}^{\mathrm{P}}{ }_{0}=0.46 \mathrm{nM}\right)$, steady-stated dissociation rates $\left(\mathrm{K}_{\mathrm{SS}}=15.4 \mathrm{nM}\right.$ and $\left.\mathrm{K}^{\mathrm{P}} \mathrm{SS}=0.49 \mathrm{nM}\right)$, and first-order elimination rates of complexes $\left(\mathrm{k}_{\text {int }}^{\mathrm{C}}=0.17 \mathrm{day}^{-1}\right.$ and $\left.\mathrm{k}^{\mathrm{P}}{ }_{\text {int }}=0.0079 \mathrm{day}^{-1}\right)$. This model showed slower turnover of targets and infliximab-TNF complex elimination rate in peripheral compartment than in central compartment. This study allowed a better understanding of the multi-scale target-mediated pharmacokinetics of infliximab. This model could be useful to improve model-based therapeutic drug monitoring of infliximab in IBD patients.
\end{abstract}

Keywords: infliximab; pharmacokinetics; target-mediated drug disposition; inflammatory bowel diseases

\section{Introduction}

Infliximab is a chimeric monoclonal antibody $(\mathrm{mAb})$ binding to tumor necrosis factor alpha (TNF- $\alpha$ ), approved in chronic inflammatory diseases both in rheumatology-e.g., rheumatoid arthritis (RA) and ankylosing spondylitis (AS) — and in inflammatory bowel diseases (IBD) — e.g., Crohn's disease (CD) or ulcerative colitis (UC). The administration of weight-adjusted infliximab doses leads to highly variable infliximab concentrations. This variability is relevant since infliximab concentrations were shown to be associated with clinical efficacy, especially in IBD [1]. Infliximab pharmacokinetics was analyzed using compartmental modeling in more than 30 studies to date. Some of these studies reported 
predictive models suitable for model-based therapeutic drug monitoring of infliximab [2-4]. The aim of individual dosing regimen to maintain serum trough concentrations of infliximab above target concentrations associated with good clinical response. In IBD, these target concentrations were reported to be $3-5 \mathrm{mg} / \mathrm{L}$ at steady-state [3].

Infliximab binds to TNF- $\alpha$ with high affinity [5-7]. This leads to the formation of infliximab-TNF complexes that are cleared by the immune system and to a mutual consumption of infliximab and TNF- $\alpha$. Therefore, to allow a good clinical response, infliximab should be in stoichiometric excess compared to TNF- $\alpha$ during all over the time of treatment. This is the case for blood TNF- $\alpha$, since infliximab concentrations are more than 10,000-fold higher than TNF- $\alpha$ levels [8-10]. However, a recent study from our group suggested that antigen mass (i.e., total amount of TNF- $\alpha$ able to interact with infliximab) is more than 200 fold higher than circulating TNF- $\alpha$ and that trough infliximab serum concentrations above target values do not lead to sustained TNF- $\alpha$ inhibition [11]. In IBD, since this phenomenon is not associated with systematic loss of response, and that TNF- $\alpha$ reservoir is admitted to be both circulating and expressed on intestine inflammatory cells (monocytes, macrophages) [12-14], it may be hypothesized that infliximab's effect is related to its binding to TNF- $\alpha$ in a 'deep' compartment.

The joint kinetics of both infliximab and target may be described using target-mediated drug disposition (TMDD) models $[15,16]$. Up to date, five publications reported targetmediated pharmacokinetics of infliximab in IBD [11,17-20]. However, none of them allowed the description of the interactions of infliximab and TNF- $\alpha$ in the deep compartment. Indeed, four of these studies used one-compartment pharmacokinetic models [11,18-20], while one used a two-compartment TMDD model which described interactions between infliximab and circulating TNF- $\alpha$ levels in the central compartment [17].

Therefore, the present study aimed at describing infliximab target-mediated pharmacokinetics in IBD patients, allowing the quantification of infliximab interactions with TNF- $\alpha$ in both central and peripheral compartments using population TMDD modeling.

\section{Methods}

\subsection{Data}

The present study was conducted using two cohorts of patients:

- $\quad$ A bicentric study of 26 ankylosing spondylitis (AS) patients (SPAXIM, NCT00607403). Inclusion and exclusion criteria were previously described [21]. Patients were treated with infliximab $5 \mathrm{mg} / \mathrm{kg}$ infusions at weeks $0,2,6,12$, and 18; blood samples were collected to measure infliximab concentrations before, 2 and $4 \mathrm{~h}$ after each infusion, and at each intermediate visit at weeks 1, 3, 4, 5, 8, 10, and 14 [21]. One patient was not assessed in the present work because he developed anti-drug antibodies (ADA) starting from the second infliximab administration;

- A retrospective cohort of 133 routine IBD patients treated with infliximab between 2006 and 2012 in the Tours University Hospital (Tours, France). For these patients, individual results were interpreted, sent to the prescriber and discussed in clinicbiological rounds. Infliximab concentrations were therefore not sought for this study and were already used in previous publications [22,23]. This cohort included patients with trough and peak infliximab concentrations measured during treatment initiation and in whom anti-drug antibodies (ADA) were not detected at least within the three first infliximab cycles. Patients were excluded if less than three concentration values were available (which excluded seven patients of our database between 2006 and 2012), if no peak concentrations were available (which excluded two more patients) and if ADA were detected at first, second of third cycles (which excluded three more patients). Of note, if ADA were detected starting for the fourth cycle, patients were not excluded from analysis, but data regarding cycles with ADA were discarded (eight patients).

Infliximab concentrations were measured using a validated enzyme-linked immunosorbent assay (ELISA). Detection, and lower and upper quantitation limits were 0.031, 0.103, 
and $15 \mathrm{mg} / \mathrm{L}()$, respectively. This assay was shown to measure unbound infliximab concentrations [24]

\subsection{Model Development}

\subsubsection{Structural Model Design}

A double TMDD model with quasi-steady-state (QSS) approximation $[25,26]$ accounting for interactions of infliximab with TNF in both central and peripheral compartments in IBD patients was developed (Figure 1, Appendix A). As in our previous study, targetmediated pharmacokinetic parameters of IBD were obtained using AS as a reference of linear pharmacokinetics. Indeed, no target influence was detected in AS despite a dense sampling protocol [27]. Levels of TNF- $\alpha$ were not measured and considered as latent variable. The double QSS model was

$$
\begin{gathered}
\frac{d C_{T}}{d t}=\operatorname{In}(t)-\frac{C L}{V_{1}} \cdot C-\frac{Q}{V_{1}} \cdot C+\frac{Q}{V_{2}} \cdot C_{P}-k_{\text {int }}^{C} \cdot\left(C_{T}-C\right) \\
\frac{d R_{T}^{C}}{d t}=k_{\text {in }}^{C}-k_{\text {out }} \cdot\left(R_{T}^{C}-C_{T}+C\right)-k_{\text {int }}^{C} \cdot\left(C_{T}-C\right) \\
C=\frac{1}{2}\left[\left(C_{T}-R_{T}^{C}-K_{S S}^{C}\right)+\sqrt{\left(C_{T}-R_{T}^{C}-K_{S S}^{C}\right)^{2}-4 \cdot K_{S S}^{C} \cdot C_{T}}\right] \\
\frac{d C_{P T}}{d t}=\frac{Q}{V_{1}} \cdot C-\frac{Q}{V_{2}} \cdot C_{P}-k_{\text {int }}^{P} \cdot\left(C_{P T}-C_{P}\right) \\
C_{P}=\frac{1}{2}\left[\left(C_{P T}^{P}-R_{T}^{P}-K_{S S}^{P}\right)+\sqrt{\left(C_{P T}-R_{T}^{P}-K_{S S}^{P}\right)^{2}-4 \cdot K_{S S}^{P} \cdot C_{P T}}\right]
\end{gathered}
$$

where $\operatorname{In}(t)$ is infliximab input function; $C$ and $C_{T}$ are unbound and total infliximab concentrations in central compartment, respectively; $C_{P}$ and $C_{P T}$ are unbound and total infliximab concentrations in peripheral compartment, respectively; $V_{1}$ and $V_{2}$ are central and peripheral volumes of distribution, respectively; $C L$ and $Q$ are systemic and intercompartmental clearances, respectively; $R_{T}^{C}$ and $R_{T}^{P}$ are latent total TNF- $\alpha$ levels interacting with infliximab in central and peripheral compartments, respectively; $k_{i n}^{C}$ and $k_{i n}^{P}$ are zero-order TNF- $\alpha$ input relative to central and peripheral compartments, respectively; $K_{S S}^{C}$ and $K_{S S}^{P}$ are steadystate dissociation constants relative to central and peripheral compartments, respectively; $k_{i n t}^{C}$ and $k_{\text {int }}^{P}$ are first-order elimination rate constants of infliximab-TNF complexes; and $k_{\text {out }}$ is first-order TNF- $\alpha$ elimination rate constant. Rather than $k_{i n}^{C}$ and $k_{i n}^{P}$, baseline levels in central $\left(R_{0}^{C}=k_{\text {in }}^{C} / k_{\text {out }}\right)$ and peripheral $\left(R_{0}^{P}=k_{\text {in }}^{P} / k_{\text {out }}\right)$ compartments were estimated. Pharmacokinetic data were analyzed using the nonlinear mixed-effect modeling software MONOLIX Suite 2020 (Lixoft ${ }^{\circledR}$, Antony, France). A large number of iterations (100 and 400 iteration kernels 1 and 2, respectively) and five Markov chains were used. Objective function (-2LL) and Fisher information matrix were computed using importance sampling and stochastic approximation methods, respectively. All parameters were estimated simultaneously. The structural target-mediated pharmacokinetic model of infliximab was developed in four steps:

1. Bicompartimental: two-compartment model with no target interaction;

2. Central TMDD: two-compartment model with target interaction in central compartment;

3. Peripheral TMDD: two-compartment model with target interaction in peripheral compartment;

4. Double TMDD: two-compartment model with target interaction in both central and peripheral compartments. 


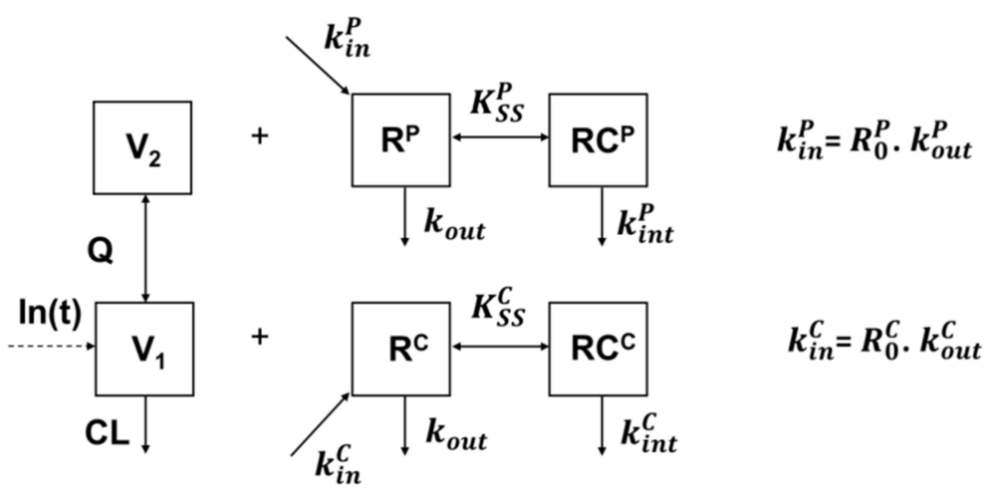

Figure 1. Target-mediated drug disposition (TMDD) with quasi-equilibrium (QSS) approximation. Base parameters are estimated for inflammatory bowel disease (IBD) and ankylosing spondylitis (AS), the latter being used as a reference, while TMDD parameters are estimated for central (below, " $\mathrm{C}$ " exponent) and peripheral (above, " $\mathrm{P}$ " exponent). $\operatorname{In}(t)$ is infliximab input function, V1 and V2 are central and peripheral volumes of distribution, respectively, CL and $Q$ are endogenous and intercompartment clearances, respectively, $\mathrm{R}_{0}, k_{i n}$, and $\mathrm{k}_{\text {out }}$ are baseline TNF- $\alpha$ amount, zero-order unbound target production rate constant and first-order destruction rate constant, respectively, $\mathrm{K}_{\mathrm{SS}}$ and $\mathrm{k}_{\mathrm{int}}$ are steady-state dissociation constant and infliximab-TNF complex elimination rate constant, respectively.

Early attempts showed that some parameters could not be estimated and had to be fixed; best strategy was to fix the value of $k_{\text {out }}$. Several values were derived from serum half-life estimations in humans (40.6 day ${ }^{-1}$ [28]), rhesus monkeys (10 or 66 day $^{-1}$ [29]), rats (33 or 154 day $^{-1}$ [30]), and rabbits (166 day ${ }^{-1}$ [31]). Based on these possible values, the double QSS model was run while fixing $k_{\text {out }}$ at the following values: 5, 10, 20, 40, 100, 150 , or $200 \mathrm{day}^{-1}$. The value leading to best model performances was retained. This was done on the central TMDD model and verified on the double TMDD model while fixing the same $\mathrm{k}_{\text {out }}$ value for both central and peripheral compartments.

\subsubsection{Statistical Models}

- Interindividual and error models

Statistical model for interindividual variability was exponential, where interindividual variances were fixed to 0 if relative standard errors (RSE) and/or shrinkage values were high. Error model was mixed additive-proportional as in our previous analyses of these data $[21,22]$.

- Influence of covariates

Covariates that were considered in this analysis were those which were found significantly associated with pharmacokinetic parameter in previous studies involving these databases - i.e., body weight (BW), sex (SX), and underlying disease [3,21,22,32]. The continuous covariate BW coded as a power function and centered on its median, whereas categorical covariates (CAT) were SX and underlying IBD disease (DIS = CD or UC); IBD was tested only on IBD patients. The influence of CAT was implemented as: $\ln \left(\theta_{\mathrm{TV}}\right)=\ln \left(\theta_{\mathrm{CAT}=0}\right)+\beta_{\mathrm{CAT}=1}$, where $\theta_{\mathrm{TV}}$ is typical value of structural parameter $\theta$, $\theta_{\mathrm{CAT}=0}$ is the value of $\theta$ for the reference category, and $\beta_{\mathrm{CAT}=1}$ is a parameter leading to the value for the other category. References for SX and DIS were females, and CD, respectively. The association of covariates with parameters was tested only with parameters for which interindividual variances was estimable.

\subsubsection{Model Evaluation}

- Model comparison

Structural and statistical models were compared using objective functions. The best structural model was the one with the lowest Akaike's information criterion (AIC). This 
criterion combines the $-2 \cdot \ln$ likelihood $(-2 \mathrm{LL})$ and twice the number of parameters to be estimated. Statistical (interindividual, convariate) models were compared using the likelihood ratio test (LRT), where the difference in -2LL between nested models was assumed to follow a $\chi^{2}$ distribution. The association of covariate with parameter distribution was assessed in two steps. First, a univariate step where the association of each covariate on parameter was tested separately. Covariates significantly associated with parameters $(\alpha<0.05)$ were added in the full model. Second, a multivariate step was made, where covariates of the full model were individually removed from the full model (backward stepwise procedure). Covariates were kept in the final model if their removal resulted in a significant increase in -2LL $(\alpha<0.02)$.

- Model goodness of fit

Models were evaluated graphically using goodness-of-fit diagnostic plots: observed vs. population (PRED) and individual (IPRED) fitted concentrations; population (WRES) and individual (IWRES) weighted residuals vs. PRED and IPRED, respectively. Visual predictive checks and normalized prediction distribution errors (NPDE) were performed by simulating 1000 replicates using both fixed and random parameters of the final model.

\subsection{Simulations}

The objective of simulations was to evaluate TNF- $\alpha$ blockade, which was quantified using total $\left(R_{T}^{C}\right.$ and $\left.R_{T}^{P}\right)$, unbound $\left(R^{C}\right.$ and $\left.R^{P}\right)$ target levels, as well as ratios of unbound/total target in central $\left(R^{C} / R_{T}^{C}\right)$ and peripheral $\left(R^{P} / R_{T}^{P}\right)$ compartments, respectively. Structural and interindividual parameter values of the final model were used to simulate median and $90 \%$ prediction intervals of infliximab concentrations and target kinetics in both compartments. Simulated dosing regimen was $5 \mathrm{mg} / \mathrm{kg}$ infused at weeks $0,2,6,14$, and 22. Ratios of 50/50 and 5/1 for male/female and CD/UC were assumed. Distribution of BW was assumed as Gaussian with mean and standard deviation of $66 \mathrm{~kg}$ and $15 \mathrm{~kg}$, respectively, with values restricted within the population range (41-110). Simulations were made using Simulx 2020 (Lixoft $^{\circledR}$, Antony, France).

\section{Results}

\subsection{Base Model}

A total of 1333 infliximab concentrations were available in the assessed 158 patients (Table 1, Figure S1). The double TMDD model allowed the best description of concentrationtime data since it led to the deepest decrease in AIC (Table 2). Optimal fixed value for $k_{\text {out }}$ was 20 day $^{-1}$ (supplemental material part 2, Table S1). All structural, interindividual and residual parameters of base and final model were estimated with good accuracy (Table 2), including $\mathrm{V}_{1}, \mathrm{~V}_{2}, \mathrm{CL}, R_{0}^{C}$, and $R_{0}^{P}$. Interindividual variances of other parameters were poorly identifiable and were set to 0 . Diagnostic plots (Figure 2) showed a good agreement between observed and model-fitted concentrations. Residuals, VPCs and NPDEs displayed neither bias nor model misspecification (Figure 2). Endogenous (i.e., non-target-mediated) elimination half-life $\left(\mathrm{T} \frac{1}{2}-\beta\right)$ was approximately 17 days.

Table 1. Summary of patient characteristics.

\begin{tabular}{lcc}
\hline \multicolumn{1}{c}{ Characteristics } & AS Cohort & IBD Cohort \\
\hline Number of patients & 25 & 133 \\
Number of samples & 488 & 845 \\
Starting dose (mg) & $400(300-400)$ & $300(280-370)$ \\
Body weight (kg) & $75(65-85)$ & $64(56-72)$ \\
Age (years) & $43(35-52)$ & $34(25-41)$ \\
Sex (females/males) & $6 / 19$ & $53 / 80$ \\
Ankylosing spondylitis & 25 & - \\
Crohn's disease & - & 108 \\
Ulcerative colitis & - & 25 \\
\hline
\end{tabular}


Table 2. Parameter estimates for different base models and final model.

\begin{tabular}{|c|c|c|c|c|c|c|c|c|c|c|c|}
\hline \multirow{3}{*}{ Parameter } & \multirow{3}{*}{ Unit } & \multicolumn{10}{|c|}{ Model } \\
\hline & & \multicolumn{2}{|c|}{ Base 2 Compartments } & \multicolumn{2}{|c|}{ Base TMDD Peripheral } & \multicolumn{2}{|c|}{ Base TMDD Central } & \multicolumn{2}{|c|}{$\begin{array}{c}\text { Base TMDD Central + } \\
\text { Peripheral }\end{array}$} & \multicolumn{2}{|c|}{$\begin{array}{c}\text { Final TMDD Central + } \\
\text { Peripheral }\end{array}$} \\
\hline & & Estimate & $\mathrm{RSE} \%$ & Estimate & RSE\% & Estimate & RSE\% & Estimate & RSE $\%$ & Estimate & RSE $\%$ \\
\hline $\mathrm{V}_{1}$ & $\mathrm{~L}$ & 3.0 & 2.4 & 2.8 & 2.6 & 2.9 & 2.5 & 2.8 & 2.5 & 2.6 & 3.3 \\
\hline $\mathrm{CL}$ & L.day $^{-1}$ & 0.29 & 7.9 & 0.28 & 4.0 & 0.18 & 7.1 & 0.20 & 6.7 & 0.16 & 9.3 \\
\hline $\mathrm{V}_{2}$ & $\mathrm{~L}$ & 2.2 & 6.9 & 1.8 & 8.3 & 1.9 & 9.8 & 1.9 & 11 & 1.9 & 8.8 \\
\hline $\mathrm{Q}$ & L.day $^{-1}$ & 0.29 & 5.1 & 1.6 & 6.6 & 0.30 & 7.8 & 1.5 & 2.2 & 1.8 & 2.0 \\
\hline $\mathrm{K}^{\mathrm{C}} \mathrm{SS}$ & $\mathrm{nM}$ & - & - & - & - & 13.5 & 8.8 & 13.7 & 16 & 15.4 & 21 \\
\hline $\mathrm{R}_{0}^{\mathrm{C}_{0}}$ & $\mathrm{nM}$ & - & - & - & - & 7.2 & 21 & 2.6 & 21 & 3.3 & 28 \\
\hline $\mathrm{k}^{\mathrm{C}}{ }_{\text {int }}$ & day $^{-1}$ & - & - & - & - & 0.089 & 11 & 0.13 & 17 & 0.17 & 11 \\
\hline $\mathrm{K}_{\mathrm{SS}}^{\mathrm{P}}$ & $\mathrm{nM}$ & - & - & 0.49 & 5.5 & - & - & 0.45 & 5.1 & 0.49 & 11 \\
\hline $\mathrm{R}^{\mathrm{P}}{ }_{0}$ & $\mathrm{nM}$ & - & - & 0.68 & 25 & - & - & 0.30 & 9.2 & 0.46 & 22 \\
\hline $\mathrm{k}^{\mathrm{P}}{ }_{\text {int }}$ & day $^{-1}$ & - & - & 0.013 & 37 & - & - & 0.0050 & 33 & 0.0079 & 36 \\
\hline $\mathrm{k}_{\text {out }}$ & day $^{-1}$ & - & - & 20 & (fixed) & 20 & (fixed) & 20 & (fixed) & 20 & (fixed) \\
\hline BW_V $V_{1}$ & - & - & - & - & - & - & - & - & - & 0.33 & 35 \\
\hline SX_V $V_{1}$ & - & - & - & - & - & - & - & - & - & 0.13 & 40 \\
\hline SX_CL & - & - & - & - & - & - & - & - & - & 0.36 & 26 \\
\hline $\mathrm{UC} \_\mathrm{R}_{0}$ & - & - & - & - & - & - & - & - & - & 0.57 & 47 \\
\hline$\omega_{\mathrm{V} 1}$ & - & 0.28 & 6.8 & 0.29 & 6.8 & 0.26 & 7.0 & 0.29 & 6.8 & 0.27 & 7.0 \\
\hline$\omega_{\mathrm{CL}}$ & - & 0.46 & 6.2 & 0.50 & 7.0 & 0.38 & 15 & 0.38 & 13.0 & 0.35 & 13 \\
\hline$\omega_{\mathrm{V} 2}$ & - & 0.62 & 9.8 & 0.32 & 24 & 0.71 & 12 & 0.36 & 24 & 0.39 & 25 \\
\hline$\omega_{\mathrm{RC} 0}$ & - & - & - & - & - & 0.98 & 15 & 1.0 & 14 & 1.0 & 15 \\
\hline$\omega_{\mathrm{RP} 0}$ & - & - & - & 0.31 & 6.8 & - & - & 1.2 & 20 & 1.1 & 16 \\
\hline$\sigma_{\text {add }}$ & $\mathrm{mg} / \mathrm{L}$ & 1.8 & 10 & 1.8 & 11 & 1.9 & 10 & 1.8 & 9.8 & 1.8 & 9.8 \\
\hline$\sigma_{\text {prop }}$ & - & 0.20 & 3.1 & 0.20 & 3.4 & 0.20 & 3.2 & 0.20 & 3.0 & 0.20 & 3.0 \\
\hline$-2 \mathrm{LL}$ & - & $10,870.99$ & - & $10,826.96$ & - & $10,818.28$ & - & $10,793.76$ & - & $10,750.05$ & - \\
\hline AIC & - & $10,888.99$ & - & $10,852.96$ & - & $10,844.28$ & - & $10,827.76$ & - & $10,792.05$ & - \\
\hline
\end{tabular}

Legends. TMDD: target-mediated drug disposition; $\mathrm{V}_{1}, \mathrm{~V}_{2}$ : central and peripheral volumes of distribution; $\mathrm{CL}$, $\mathrm{Q}:$ systemic and intercompartment clearances; $\mathrm{K}_{\mathrm{SS}}$ : steady-state dissociation constants relative to central and peripheral compartments; $\mathrm{R}_{0}$ : baseline TNF- $\alpha$ amount relative to central and peripheral compartments; $\mathrm{k}_{\text {int }}$ : infliximab-TNF- $\alpha$ complex elimination rate constant, " $\mathrm{C}$ " and " $\mathrm{P}$ " stand for central and peripheral compartments, respectively; $\mathrm{k}_{\text {out }}$ : TNF- $\alpha$ elimination rate constant; $t \mathrm{BW}$ : body weight; SX: sex; UC: ulcerative colitis;

$-2 \mathrm{LL}:-2$ ln-likelihood; AIC: Akaike's information criterion.
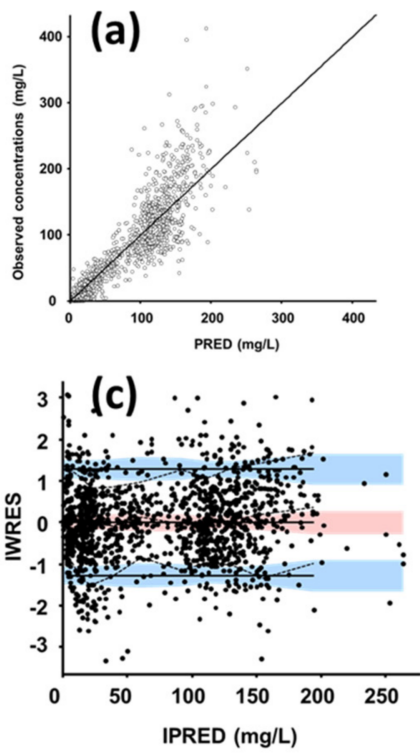

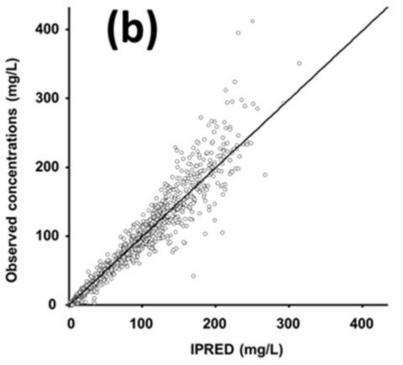

(d)

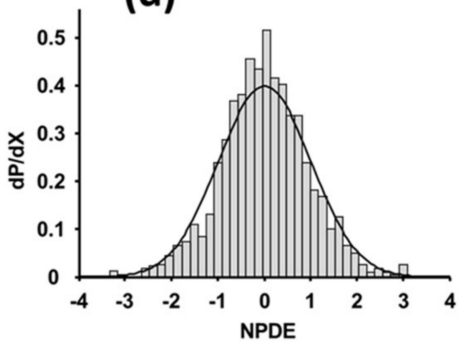

(e)

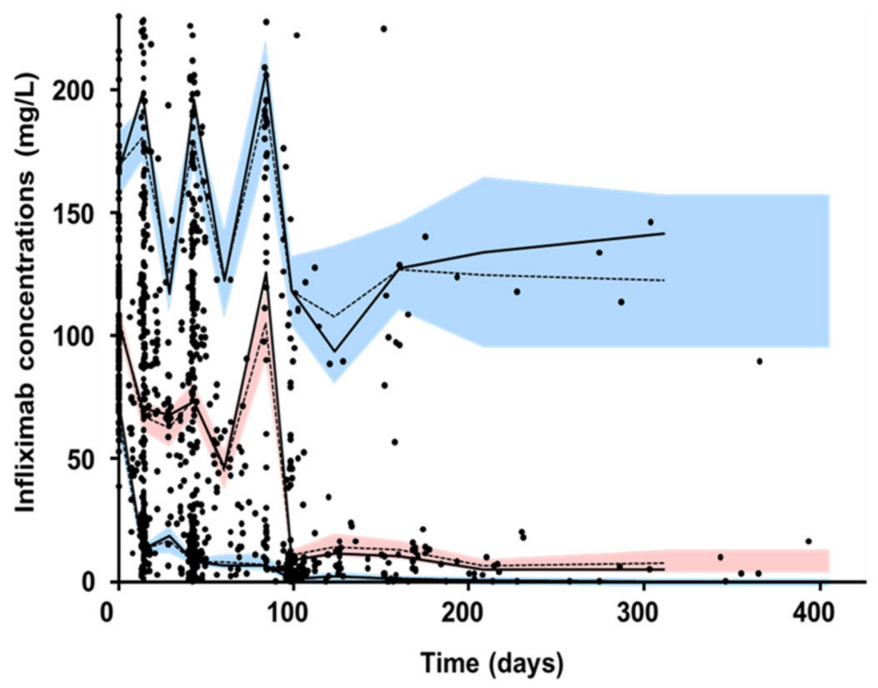

Figure 2. Diagnostic plots of the final pharmacokinetic TMDD quasi-steady-state (QSS) model. (a) Observed vs. population model fitted infliximab concentrations (PRED) and (b) observed vs. individual model-fitted infliximab concentrations, open circles are observed vs. fitted concentrations and line is first bisector; (c) individual weighted residuals (IWRES) vs. IPRED; black circles are IWRES vs. IPRED; (d) normalized prediction distribution error (NPDE) distribution vs. Gaussian probability density function; dashed line is theoretical Gaussian distribution; (e) visual predictive check; observed concentration (black circles), theoretical (dashed lines), and empirical (continuous lines) percentiles (from bottom to top: 10\%,50\% and 100\% percentiles) and prediction interval (from bottom to top: $10 \%, 50 \%$, and $90 \%$ prediction intervals. 


\subsection{Final Model}

During the univariate step, BW and SX significantly influenced both $\mathrm{V}_{1}$ and CL, while IBD significantly influenced $R_{0}^{C}$. Multivariate backward stepwise step confirmed increased $\mathrm{V}_{1}$ with increasing BW (LRT $\left.=8.11, p=0.0044\right)$ and in males $\left(\mathrm{V}_{1 \text {,males }}=2.8 \mathrm{~L}\right.$, $\mathrm{LRT}=6.17, p=0.013)$, while $\mathrm{CL}$ was increased in males $\left(\mathrm{CL}_{\text {males }}=0.23 \mathrm{~L} /\right.$ day, $\mathrm{LRT}=15.29$, $p=9.2 \times 10^{-5}$ ). In addition, UC was associated with increased $R_{0}^{C}$ compared to CD $\left(R_{0}^{C}=5.8 \mathrm{nM}, \mathrm{LRT}=5.71, p=0.017\right)$.

\subsection{Simulations}

In simulations of $90 \%$ intervals of infliximab concentrations, total and unbound target levels and unbound/total target level ratios $\left(R / R_{T}\right)$ showed substantial differences between central and peripheral compartments and a large interindividual variability. Notably, the turnover of targets in peripheral compartment was slower than in the central compartment. As a result, before third and fourth infliximab infusions, while $R^{C} / R_{T}^{C}$ ratio re-increases above $30 \%$ in median, $R^{P} / R_{T}^{P}$ ratio remained at less than $3 \%$, with a large interindividual variability (Figure 3). An infliximab serum concentration of $5 \mathrm{mg} / \mathrm{L}$ was associated with median $R^{C} / R_{T}^{C}$ and $R^{P} / R_{T}^{P}$ ratios of $26 \%$ and $1.2 \%$, respectively. Since elimination half-life of infliximab is much higher than that of TNF- $\alpha$, the amount maximum of total target is higher (10- to 100-fold) than baseline target amount.

\section{Concentrations $(\mathrm{mg} / \mathrm{L})$}
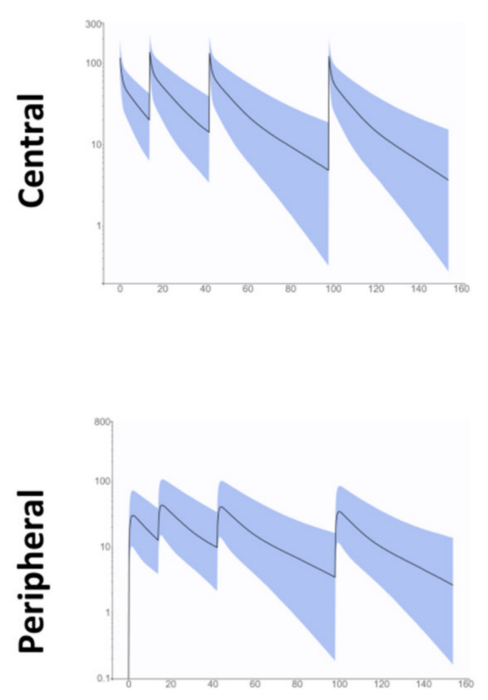

Total target (nM)
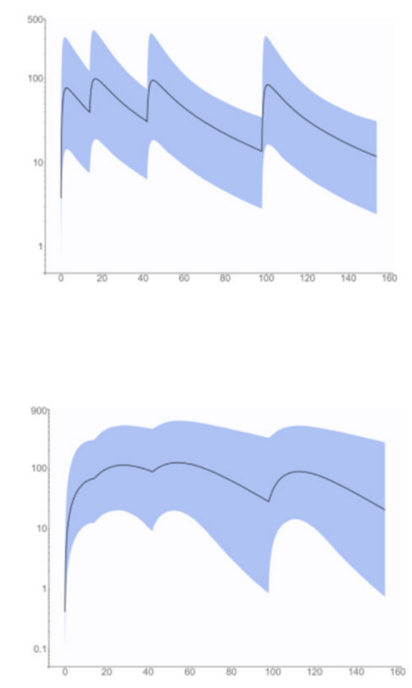

Unbound target (nM)
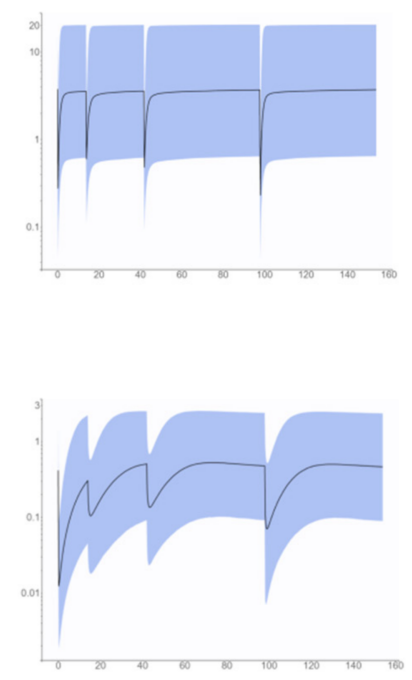

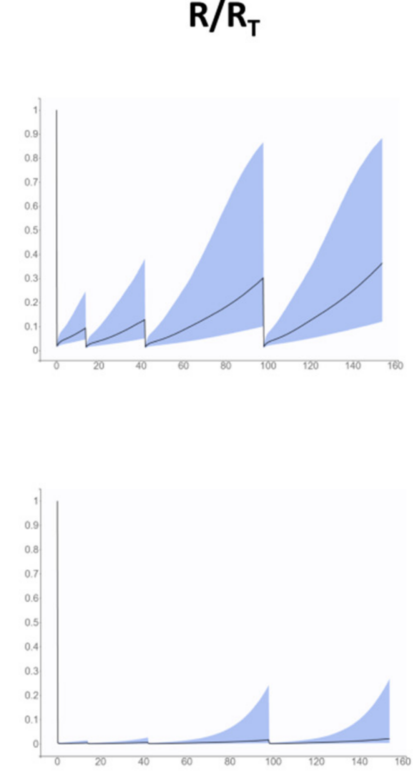

$\mathbf{R} / \mathbf{R}_{\mathrm{T}}$

Figure 3. From left to right, $90 \%$ prediction intervals for infliximab concentrations, total target and unbound target levels, and unbound/total target ratio relative to central (above) and peripheral (below) compartments. Blue shaded are $90 \%$ prediction intervals and line is median profile.

\section{Discussion}

To our knowledge, this is the first study that investigated target-mediated elimination of infliximab in both central and peripheral compartments in inflammatory bowel diseases (IBD) using a double target-mediated drug disposition (TMDD) model. We showed that TNF- $\alpha$ turnover and its interactions with infliximab (infliximab-TNF complex formation and clearance) were substantially different between these two compartments.

Up to date, infliximab pharmacokinetics was studied using compartmental modeling in 36 publications, including 22 that used population two-compartment models, of which 15 in IBD patients [32,33]. The influence of target antigen on infliximab pharmacokinetics was suggested in several publications using covariates related to inflammation (CRP levels, fecal calprotectin, erythrocyte sedimentation rate) [16,32], and described using TMDD 
models in six studies, of which four were made on aggregated data [18-20,34], with two using population modeling [11,17].

As in our previous work, our estimates of baseline target levels $\left(\mathrm{R}_{0}\right)$ in central $(3.3 \mathrm{nM})$ and peripheral $(0.46 \mathrm{nM})$ compartment were dramatically greater than circulating TNF- $\alpha$ levels (0.000038 nM [17]). As discussed in our previous publication [11], this suggests that our model measures antigen mass, both inside and outside the bloodstream. Nevertheless, the total antigen mass (i.e., central + peripheral) may be even higher: indeed, the present estimation is more than 10 -fold that of our previous work $(0.46 \mathrm{nM})$ and should be more accurate since our previous study was made using a one-compartment model. This suggests that most of TNF- $\alpha$ targeted by infliximab in the central compartment is not circulating in the bloodstream.

Moreover, our results suggest that infliximab-TNF- $\alpha$ interactions are very different in central and peripheral compartments. Notably, the elimination rate constant of infliximabTNF- $\alpha$ complexes is 20 -fold higher in central $\left(0.17\right.$ day $\left.^{-1}\right)$ than in peripheral $\left(0.0079\right.$ day $\left.^{-1}\right)$ compartment, suggesting a dramatically slower elimination of complexes and therefore a longer retention of infliximab in peripheral compartment. The value of central $k_{\text {int }}$ is in agreement with median value reported for mAbs studied using TMDD modeling (0.13 day ${ }^{-1}$ [16]) but still inferior to the value estimated by Berends et al. $\left(0.98\right.$ day $^{-1}$ [17]). Indeed, this latter study quantified a rapid elimination of complexes due to blood circulating TNF- $\alpha$, while the present, as well as previous ones [11,18-20], may have quantified a slower kinetics of complexes involving the whole antigen mass.

The difference in steady-state dissociation constant $\left(\mathrm{K}_{\mathrm{SS}}\right)$ estimates between central $(15.4 \mathrm{nM})$ and peripheral $(0.49 \mathrm{nM})$ compartments may be linked to target turnover and expression as well as to differences in infliximab-TNF affinities between central and peripheral compartment. In the central compartment, our $\mathrm{K}_{\mathrm{SS}}$ estimate is similar to that reported by Berends et al. (14 $\mathrm{nM} \mathrm{[17]),} \mathrm{while} \mathrm{that} \mathrm{of} \mathrm{the} \mathrm{peripheral} \mathrm{compartment} \mathrm{is} \mathrm{similar}$ to values linked to slow kinetics of complexes reported in previous publications on IBD patients from Kimura et al. (0.468 nM, [20]) and us (0.45 nM [11]). Of note, these latter values were dissociation constants $\left(\mathrm{K}_{\mathrm{D}}\right)$, but they should be similar to $\mathrm{K}_{\mathrm{SS}}$ values because they were associated with low values of $k_{\text {int }}[26,35]$. The reasons of the 30 -fold ratio between central and peripheral $\mathrm{K}_{\mathrm{SS}}$ values are unclear. A possible reason might linked to the existence of two forms of TNF- $\alpha$-i.e., homotrimers or monomers, trimeric TNF- $\alpha$ binding to receptors TNF receptors 1 and 2 and therefore the active form [36]. Trimers are known to monomerize for low concentrations [37], re-trimerization occurring if monomer concentrations are sufficient $(>10 \mathrm{nM})$ [38]. Thus, low concentrations of circulating TNF- $\alpha$ are in favor of predominant monomer form in blood. Besides, concentrations of trimeric TNF- $\alpha$ are increased locally due to the presence of receptors [39] and due to anti-TNF, as infliximab, which stabilize trimeric TNF- $\alpha$ [40]. Trimeric TNF- $\alpha$ leads to the formation of infliximab-TNF complexes involving two or three molecules of each which are hypothesized to be more stable than simple 1:1 complexes [41]. Hence, the lower dissociation constant in peripheral compartment might be due to more stable complexes formed with trimeric than monomeric TNF- $\alpha$.

It is generally considered that an efficient dosing strategy for mAbs should lead to sufficient target blockade, a variable which is often evaluated using the unbound/total target ratio $\left(R / R_{T}\right)[16,42]$. Previous works made by Berends et al. [17] and us [11] showed that infliximab treatment was not associated with sustained TNF- $\alpha$ blockade, despite an absence of systematic loss of response. In our previous work [11], we had hypothesized a multi-scale turnover of TNF- $\alpha$ in IBD patients, including the existence of a deep compartment in which kinetics differed from that of the central compartment and which had still not been quantified. This compartment may be linked to TNF- $\alpha$ expressed on intestine inflammatory cells (monocytes, macrophages). The present work not only confirms that TNF- $\alpha$ is not durably occupied in the central compartment, but also suggests an almost full TNF- $\alpha$ blockade in peripheral compartment of most patients. This phenomenon sug- 
gests that sustained clinical response may be due to target blockade on inflammatory intestine cells.

Interestingly, our double TMDD model displays an apparent tri-phasic decay of infliximab concentrations in serum (Concentrations in central compartment, Figure 3), which suggests that infliximab pharmacokinetics in IBD patients is more complex than what was previously described. Indeed, estimates of two-compartment parameters varied markedly between studies, notably those of peripheral compartment. Notably, across the 15 publications of two-compartment kinetics of infliximab, there is a large disparity in estimates of intercompartment clearance (Q) [33]. Due to low values of $Q$ (less than $0.1 \mathrm{~L} /$ day in average), one half of studies reported long distribution $\left(\mathrm{T} \frac{1}{2}-\alpha>3.5\right.$ days) and elimination ( $T \frac{1}{2}-\beta>18$ days) half-lives, while pharmacokinetic studies conducted with dense datasets reported high values of $Q$ (3.7 L/day in average). Similarly, in the present work, values of $Q$ were lower if no peripheral TMDD compartment was included ( $0.3 \mathrm{vs.}$ $1.5 \mathrm{~L} /$ day, Table 2). The discrepancies in $\mathrm{Q}$ value estimates might therefore be related to the complex pharmacokinetic behavior of infliximab.

Conversely, publications that reported long $\mathrm{T}_{2}^{1}-\alpha$ were based on two-compartment models that may have not captured the actual distribution phase, but rather the 'intermediate' phase of the apparent tri-phasic elimination shape. This may be explained not only by an over-simplistic model, but also by data paucity [32] and differences in concentration measurement techniques [2]. The large differences in pharmacokinetic parameter values between studies may be an issue for concentration forecasting in therapeutic drug monitoring [43]. In that context, our TMDD model might overcome, at least in part, this issue.

Central baseline target amount was significantly higher in UC than in CD patients. This result is consistent with our previous publication in which both volume of distribution and clearance were higher in UC than in CD [44]. This difference is difficult to explain since no clear difference in TNF- $\alpha$ expression between CD and UC was reported [8,9]. This result may be linked to our cohort and may not be representative of all IBD patients treated with infliximab.

Our study has limitations. First, our model was developed using trough and peak concentration data only, as most of infliximab pharmacokinetic studies [32,33]. This prevented us from estimating TNF- $\alpha$ elimination rate constants in central and peripheral compartments, as well as interindividual variances of some parameters $\left(Q, K_{S S}, k_{i n t}\right)$. Second, the elimination rate constant of TNF- $\alpha$ had to be fixed and assumed to be equal in both compartments. Of note, all previous infliximab TMDD models necessitated fixed values of dissociation constants $\left(K_{D}[11,18-20,34]\right.$ or $K_{S S}$ [17]). Third, as in our previous work [11], we had to use a reference dataset for which we assumed no influence of antigen mass on infliximab pharmacokinetics, i.e., AS patients [21]. Even if no influence of antigen mass has been detected [27], such an influence cannot be definitely excluded. Nevertheless, the use of AS database as a reference was possible because all concentrations were measured using the same ELISA technique [24]. Fourth, both TNF- $\alpha$ were assumed as independent, which may be oversimplistic. Unfortunately, attempts to build a two-compartment model for TNF- $\alpha$ kinetics did not provide results that overcame our double TMDD model (supplemental material part 3, Figures S2 and S3). Overall, due to these limitations, TMDD parameter estimates may have been biased and should therefore be considered with caution (supplemental material part 3, Table S2). Last but not least, no covariate linked to inflammation (CRP levels, erythrocyte sedimentation rates, fecal calprotectin [16,32]) or no clinical improvement endpoints were available in this database, which prevented us from interpreting our $\mathrm{R}_{0}$ estimates in both central and peripheral compartments and to investigate the link between TNF- $\alpha$ occupancy and clinical response.

\section{Conclusions}

This study provided the most complete description of infliximab pharmacokinetics in IBD patients. This model allowed us to take a step forward the understanding of the complexity of infliximab target-mediated pharmacokinetics which involves a multi- 
scale turnover of TNF- $\alpha$. Nevertheless, further studies are still necessary to evaluate the relevance of our description of target occupancy kinetics. Our model should be applied to existing or upcoming data sets, which would ideally provide biological and/or clinical monitoring data, allowing the description of the relationship between target kinetics (unbound target or unbound/total target ratio) and monitoring data.

Supplementary Materials: The following are available online at https: / www.mdpi.com/article / 10.3390/pharmaceutics13111821/s1, Figure S1: Spaghetti plots representing observed infliximab concentrations in time for inflammatory bowel disease patients (left) and ankylosing spondylitis patients (right). Red points are concentrations below the lower limit of quantitation $(0.103 \mathrm{mg} / \mathrm{L})$, Figure S2: Target-mediated drug disposition (TMDD) with quasi-equilibrium (QSS) approximation. This model assumes TNF- $\alpha$ input $\left(\mathrm{k}_{\text {in }}\right)$ and output $\left(\mathrm{k}_{\text {out }}\right)$ in central and peripheral compartments, respectively, and bidirectional exchanges of unbound TNF- $\alpha\left(\mathrm{k}_{\mathrm{pc}}\right.$ and $\left.\mathrm{k}_{\mathrm{cp}}\right)$. Transfer of infliximabTNF- $\alpha$ complexes is assumed to be driven by infliximab transfer kinetics $\left(\mathrm{k}_{12}\right.$ and $\left.\mathrm{k}_{21}\right)$. While elimination rate constant of complexes are supposed different in central $\left(k_{\text {int }}^{C}\right)$ and peripheral $\left(k_{\text {int }}^{P}\right)$ compartments, steady-state $\left(\mathrm{K}_{\mathrm{SS}}\right)$ dissociation constant is supposed to be of the same value in both compartments, Figure S3: Diagnostic plots of the modified TMDD model. (a) normalized prediction distribution error (NPDE) distribution vs. Gaussian probability density function; dashed line is theoretical Gaussian distribution; (b) visual predictive check; observed concentration (black circles), theoretical (dashed lines) and empirical (continuous lines) percentiles (from bottom to top: 10\%, 50\% and $100 \%$ percentiles) and prediction interval (from bottom to top: $10 \%, 50 \%$ and $90 \%$ prediction intervals, Table S1: Selection of fixed TNF- $\alpha$ elimination rate constant values, Table S2: Parameter estimates for final modified TMDD model.

Author Contributions: Conceptualization, D.T., O.L.T. and G.P.; Methodology, D.T., O.L.T. and G.P.; Software, D.T. and O.L.T.; Validation, D.T., O.L.T. and G.P.; Formal analysis, D.T. and O.L.T.; Investigation, D.T., O.L.T., C.P., T.B.-A., C.D. and G.P.; Resources: L.P., D.M. (Driffa Moussata), C.P., D.M. (Denis Mulleman), P.G. and G.P.; Data curation, D.T., C.P., C.D. and G.P.; Writing-original draft preparation: D.T. and O.L.T.; Writing-review and editing, D.T., O.L.T., T.B.-A., D.M. (Driffa Moussata), and G.P.; Visualization, D.T., O.L.T. and G.P.; Supervision; G.P.; Project administration, D.T. and G.P. All authors have read and agreed to the published version of the manuscript.

Funding: This research received no external funding.

Institutional Review Board Statement: The study was conducted according to the guidelines of the Declaration of Helsinki and approved by Ethics Committee: CPP de Tours Ouest- 1 for the SPAXIM study (NCT00607403). Inflammatory bowel disease samples were part of routine standard of care and were not kept, which did not necessitate any declaration of biological collection.

Informed Consent Statement: Informed consent was obtained from all subjects involved in the SPAXIM study (NCT00607403). Between 2006 and 2011 informed consent was not sought, while starting from 2011, non-opposition was sought, in accordance with institutional guidelines.

Data Availability Statement: The data presented in this study are available on request from the corresponding author. The data are not publicly available because belong to the University hospital of Tours who has to authorize access to the database.

Acknowledgments: The authors thank Anne-Claire Duveau and Caroline Guerineau-Brochon for technical assistance with infliximab assays; and the medical staff and nurses from the Rheumatology and Gastroenterology departments. Samples of the SPAXIM study (NCT00607403) were managed (centralization, preparation, and storage) by the Biological Resource Centre of Tours (Centre de Ressources Biologiques de Touraine) BB-0033-00013. Measurements of infliximab serum concentrations were carried out within the CePiBAc (Centre Pilote de suivi Biologique des traitements par Anticorps) platform, which was co-financed by the European Union and the European Regional Development Fund. This work was partly supported by the French Higher Education and Research Ministry under the program 'investissements d'avenir' (Grant agreement: LabEx MAbImprove ANR-10-LABX-53-01).

Conflicts of Interest: D.T. acted as a consultant and has given lectures on behalf of his institution for Amgen, Sanofi, Boehringer-Ingelheim, and Novartis. L.P. has acted as a consultant for Abbvie, Janssen-Cilag, Pfizer, and Takeda. T.B.-A. reports support for travel to congresses from Servier and 
BMS. D.M. (Denis Mulleman) has acted as a consult- ant and given lectures on behalf of his institution for Pfizer, Novartis, and Grifols; he has been invited to attend an international congress by JanssenCilag. His institution received grants for research from the non-governmental organization Lions Club Tours Val de France. P.G. reports Personal fees from AbbVie, Amgen, Biogen, BMS, Celgene, Chugai, Fresenius Kabi, Janssen-Cilag, Lilly, MSD, Novartis, Pfizer, Sanofi, UCB. G.P. has received grants for his research team from Roche Pharma, Chugai, Pfizer, Novartis, and Sanofi-Genzyme. Other authors declare no conflict of interest.

\section{Appendix A Monolix Code for Double TMDD Model}

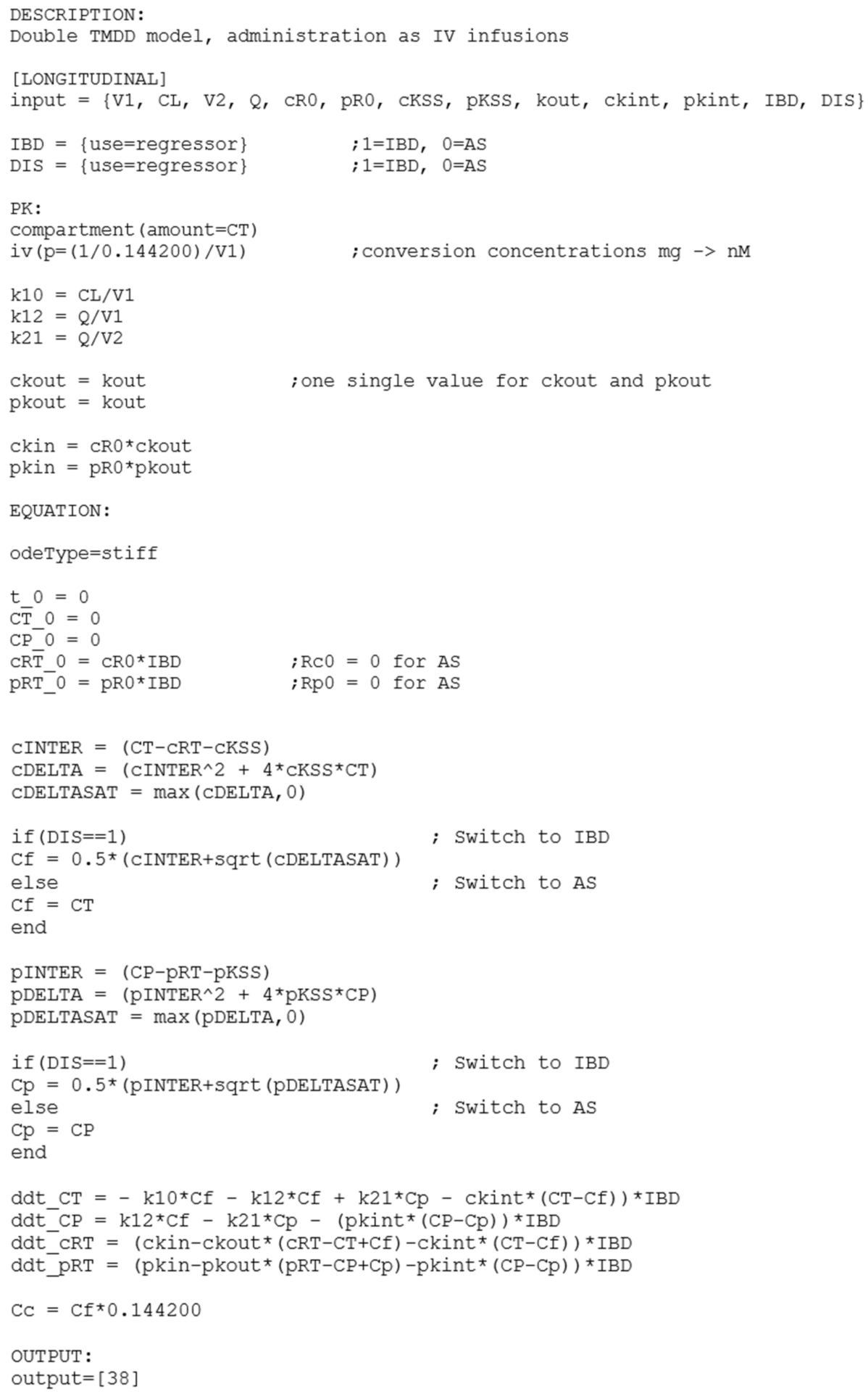

; TMDD null for AS

; RCT $=0$ for AS ; $\mathrm{RpT}=0$ for $\mathrm{AS}$ 


\section{References}

1. Paintaud, G.; Passot, C.; Ternant, D.; Bertolotto, A.; Bejan-Angoulvant, T.; Pascual-Salcedo, D.; Mulleman, D. Rationale for Therapeutic Drug Monitoring of Biopharmaceuticals in Inflammatory Diseases. Ther. Drug Monit. 2017, 39, 339-343. [CrossRef] [PubMed]

2. Eser, A.; Primas, C.; Reinisch, S.; Vogelsang, H.; Novacek, G.; Mould, D.R.; Reinisch, W. Prediction of Individual Serum Infliximab Concentrations in Inflammatory Bowel Disease by a Bayesian Dashboard System. J. Clin. Pharm. 2018, 58, 790-802. [CrossRef] [PubMed]

3. Ternant, D.; Passot, C.; Aubourg, A.; Goupille, P.; Desvignes, C.; Picon, L.; Lecomte, T.; Mulleman, D.; Paintaud, G. Model-Based Therapeutic Drug Monitoring of Infliximab Using a Single Serum Trough Concentration. Clin. Pharm. 2017, 2017, $017-0621$. [CrossRef] [PubMed]

4. Xiong, Y.; Mizuno, T.; Colman, R.; Hyams, J.; Noe, J.D.; Boyle, B.; Tsai, Y.T.; Dong, M.; Jackson, K.; Punt, N.; et al. Real-World Infliximab Pharmacokinetic Study Informs an Electronic Health Record-Embedded Dashboard to Guide Precision Dosing in Children with Crohn's Disease. Clin. Pharm. Ther. 2021, 109, 1639-1647. [CrossRef]

5. Kaymakcalan, Z.; Sakorafas, P.; Bose, S.; Scesney, S.; Xiong, L.; Hanzatian, D.K.; Salfeld, J.; Sasso, E.H. Comparisons of affinities, avidities, and complement activation of adalimumab, infliximab, and etanercept in binding to soluble and membrane tumor necrosis factor. Clin. Immunol. 2009, 131, 308-316. [CrossRef]

6. Kim, M.S.; Lee, S.H.; Song, M.Y.; Yoo, T.H.; Lee, B.K.; Kim, Y.S. Comparative analyses of complex formation and binding sites between human tumor necrosis factor-alpha and its three antagonists elucidate their different neutralizing mechanisms. J. Mol. Biol. 2007, 374, 1374-1388. [CrossRef]

7. Scallon, B.; Cai, A.; Solowski, N.; Rosenberg, A.; Song, X.Y.; Shealy, D.; Wagner, C. Binding and functional comparisons of two types of tumor necrosis factor antagonists. J Pharm. Exp. Ther. 2002, 301, 418-426. [CrossRef]

8. MacDonald, T.T.; Hutchings, P.; Choy, M.Y.; Murch, S.; Cooke, A. Tumour necrosis factor-alpha and interferon-gamma production measured at the single cell level in normal and inflamed human intestine. Clin. Exp. Immunol. 1990, 81, 301-305. [CrossRef]

9. Murch, S.H.; Lamkin, V.A.; Savage, M.O.; Walker-Smith, J.A.; MacDonald, T.T. Serum concentrations of tumour necrosis factor alpha in childhood chronic inflammatory bowel disease. Gut 1991, 32, 913-917. [CrossRef]

10. Schulz, M.; Dotzlaw, H.; Neeck, G. Ankylosing spondylitis and rheumatoid arthritis: Serum levels of TNF-Î̀ and Its soluble receptors during the course of therapy with etanercept and infliximab. BioMed Res. Int. 2014, 2014, 24. [CrossRef]

11. Ternant, D.; Pfister, M.; Le Tilly, O.; Mulleman, D.; Picon, L.; Willot, S.; Passot, C.; Bejan-Angoulvant, T.; Lecomte, T.; Paintaud, G.; et al. Infliximab Treatment Does Not Lead to Full TNF- $\alpha$ Inhibition: A Target-Mediated Drug Disposition Model. Clin. Pharm. 2021, 2021, 021-01057.

12. Buhl, S.; Dorn-Rasmussen, M.; Brynskov, J.; Ainsworth, M.A.; Bendtzen, K.; Klausen, P.H.; Bolstad, N.; Warren, D.J.; Steenholdt, C. Therapeutic thresholds and mechanisms for primary non-response to infliximab in inflammatory bowel disease. Scand. J. Gastroenterol. 2020, 55, 884-890. [CrossRef]

13. Deora, A.; Hegde, S.; Lee, J.; Choi, C.H.; Chang, Q.; Lee, C.; Eaton, L.; Tang, H.; Wang, D.; Lee, D.; et al. Transmembrane TNF-dependent uptake of anti-TNF antibodies. MAbs 2017, 9, 680-695. [CrossRef]

14. Olesen, C.M.; Coskun, M.; Peyrin-Biroulet, L.; Nielsen, O.H. Mechanisms behind efficacy of tumor necrosis factor inhibitors in inflammatory bowel diseases. Pharm. Ther. 2016, 159, 110-119. [CrossRef]

15. Mager, D.E.; Jusko, W.J. General pharmacokinetic model for drugs exhibiting target-mediated drug disposition. J. Pharm. Pharmacodyn. 2001, 28, 507-532. [CrossRef]

16. Ternant, D.; Azzopardi, N.; Raoul, W.; Bejan-Angoulvant, T.; Paintaud, G. Influence of Antigen Mass on the Pharmacokinetics of Therapeutic Antibodies in Humans. Clin. Pharm. 2019, 58, 169-187. [CrossRef]

17. Berends, S.E.; van Steeg, T.J.; Ahsman, M.J.; Singh, S.; Brandse, J.F.; D’Haens, G.; Mathôt, R.A.A. Tumor necrosis factor-mediated disposition of infliximab in ulcerative colitis patients. J. Pharm. Pharm. 2019, 46, 543-551. [CrossRef]

18. Furuya, Y.; Ozeki, T.; Takayanagi, R.; Yokoyama, H.; Okuyama, K.; Yamada, Y. Theory based analysis of anti-inflammatory effect of infliximab on Crohn's disease. Drug Metab. Pharmacokinet. 2007, 22, 20-25. [CrossRef]

19. Kimura, K.; Takayanagi, R.; Yokoyama, H.; Yamada, Y. Theory-based analysis of anti-inflammatory effect of infliximab on Crohn's disease and rheumatoid arthritis. Rheumatol. Int. 2012, 32, 145-150. [CrossRef]

20. Kimura, K.; Yoshida, A.; Katagiri, F.; Takayanagi, R.; Yamada, Y. Prediction of clinical effects of infliximab administered for inflammatory bowel disease based on pharmacokinetic and pharmacodynamic modeling. Biopharm. Drug Dispos. 2019, 40, 250-261. [CrossRef]

21. Ternant, D.; Mulleman, D.; Lauferon, F.; Vignault, C.; Ducourau, E.; Wendling, D.; Goupille, P.; Paintaud, G. Influence of methotrexate on infliximab pharmacokinetics and pharmacodynamics in ankylosing spondylitis. Br. J. Clin. Pharm. 2011, 2011, 1365-2125. [CrossRef]

22. Aubourg, A.; Picon, L.; Lecomte, T.; Bejan-Angoulvant, T.; Paintaud, G.; Ternant, D. A robust estimation of infliximab pharmacokinetic parameters in Crohn's disease. Eur. J. Clin. Pharm. 2015, 71, 1541-1542. [CrossRef]

23. Ternant, D.; Aubourg, A.; Magdelaine-Beuzelin, C.; Degenne, D.; Watier, H.; Picon, L.; Paintaud, G. Infliximab pharmacokinetics in inflammatory bowel disease patients. Ther. Drug Monit. 2008, 30, 523-529. [CrossRef]

24. Ternant, D.; Mulleman, D.; Degenne, D.; Willot, S.; Guillaumin, J.M.; Watier, H.; Goupille, P.; Paintaud, G. An enzyme-linked immunosorbent assay for therapeutic drug monitoring of infliximab. Ther. Drug Monit. 2006, 28, 169-174. [CrossRef] 
25. Dua, P.; Hawkins, E.; van der Graaf, P.H. A Tutorial on Target-Mediated Drug Disposition (TMDD) Models. CPT Pharmacomet. Syst. Pharm. 2015, 4, 324-337. [CrossRef]

26. Gibiansky, L.; Gibiansky, E. Target-mediated drug disposition model: Approximations, identifiability of model parameters and applications to the population pharmacokinetic-pharmacodynamic modeling of biologics. Expert Opin. Drug Metab Toxicol. 2009, 5, 803-812. [CrossRef]

27. Ternant, D.; Picon, L.; Cartron, G.; Mulleman, D.; Campone, M.; Merlin, J.; Goupille, P.; Büchler, M.; Lecomte, T.; Paintaud, G. Revisiting Target-Mediated Elimination of Therapeutic Antibodies: The Irreversible Binding Approximation. PAGE 28 (2019) Abstr 9169. Available online: www.page-meeting.org/ ?abstract=9169 (accessed on 18 October 2021).

28. Creaven, P.J.; Plager, J.E.; Dupere, S.; Huben, R.P.; Takita, H.; Mittelman, A.; Proefrock, A. Phase I clinical trial of recombinant human tumor necrosis factor. Cancer Chemother. Pharmacol. 1987, 20, 137-144. [CrossRef]

29. Greischel, A.; Zahn, G. Pharmacokinetics of recombinant human tumor necrosis factor alpha in rhesus monkeys after intravenous administration. J. Pharm. Exp. Ther. 1989, 251, 358-361.

30. Zahn, G.; Greischel, A. Pharmacokinetics of tumor necrosis factor alpha after intravenous administration in rats. Dose dependence and influence of tumor necrosis factor beta. Arzneimittelforschung 1989, 39, 1180-1182.

31. Beutler, B.A.; Milsark, I.W.; Cerami, A. Cachectin/tumor necrosis factor: Production, distribution, and metabolic fate in vivo. J. Immunol. 1985, 135, 3972-3977.

32. Bensalem, A.; Ternant, D. Pharmacokinetic Variability of Therapeutic Antibodies in Humans: A Comprehensive Review of Population Pharmacokinetic Modeling Publications. Clin Pharm. 2020. Online ahead of print. [CrossRef] [PubMed]

33. Le Tilly, O.; Bejan-Angoulvant, T.; Paintaud, G.; Ternant, D. Letter to Dreesen et al. on their article "Modelling of the Relationship Between Infliximab Exposure, Faecal Calprotectin, and Endoscopic Remission in Patients With Crohn's Disease"-A comprehensive review of infliximab population pharmacokinetic modelling publications. Br. J. Clin. Pharm. 2021, 2020, 14554.

34. Kimura, K.; Takayanagi, R.; Yokoyama, H.; Yamada, Y. Theory-based analysis of the anti-inflammatory effect of TNF inhibitors on rheumatoid arthritis. Drug 2014, 29, 272-277. [CrossRef] [PubMed]

35. Peletier, L.A.; Gabrielsson, J. Dynamics of target-mediated drug disposition: Characteristic profiles and parameter identification. J. Pharm. Pharm. 2012, 39, 429-451. [CrossRef]

36. Smith, R.A.; Kirstein, M.; Fiers, W.; Baglioni, C. Species specificity of human and murine tumor necrosis factor. A comparative study of tumor necrosis factor receptors. J. Biol. Chem. 1986, 261, 14871-14874. [CrossRef]

37. van Schie, K.A.; Ooijevaar-de Heer, P.; Dijk, L.; Kruithof, S.; Wolbink, G.; Rispens, T. Therapeutic TNF Inhibitors can Differentially Stabilize Trimeric TNF by Inhibiting Monomer Exchange. Sci. Rep. 2016, 6, 32747. [CrossRef]

38. Corti, A.; Fassina, G.; Marcucci, F.; Barbanti, E.; Cassani, G. Oligomeric tumour necrosis factor alpha slowly converts into inactive forms at bioactive levels. Biochem. J. 1992, 284, 905-910. [CrossRef]

39. Lim, H.; Lee, S.H.; Lee, H.T.; Lee, J.U.; Son, J.Y.; Shin, W.; Heo, Y.S. Structural Biology of the TNF- $\alpha$ Antagonists Used in the Treatment of Rheumatoid Arthritis. Int. J. Mol. Sci. 2018, 19, 768. [CrossRef]

40. Daub, H.; Traxler, L.; Ismajli, F.; Groitl, B.; Itzen, A.; Rant, U. The trimer to monomer transition of Tumor Necrosis Factor-Alpha is a dynamic process that is significantly altered by therapeutic antibodies. Sci. Rep. 2020, 10, 9265. [CrossRef]

41. Tran, B.N.; Chan, S.L.; Ng, C.; Shi, J.; Correia, I.; Radziejewski, C.; Matsudaira, P. Higher order structures of Adalimumab, Infliximab and their complexes with TNFÎ \pm revealed by electron microscopy. Protein Sci. 2017, 26, 2392-2398. [CrossRef]

42. Ait-Oudhia, S.; Ovacik, M.A.; Mager, D.E. Systems pharmacology and enhanced pharmacodynamic models for understanding antibody-based drug action and toxicity. MAbs 2017, 9, 15-28. [CrossRef]

43. Konecki, C.; Feliu, C.; Cazaubon, Y.; Giusti, D.; Tonye-Libyh, M.; Brixi, H.; Cadiot, G.; Biron, A.; Djerada, Z. External Evaluation of Population Pharmacokinetic Models and Bayes-Based Dosing of Infliximab. Pharmaceutics 2021, 13, 1191. [CrossRef]

44. Passot, C.; Mulleman, D.; Bejan-Angoulvant, T.; Aubourg, A.; Willot, S.; Lecomte, T.; Picon, L.; Goupille, P.; Paintaud, G.; Ternant, D. The underlying inflammatory chronic disease influences infliximab pharmacokinetics. MAbs 2016, 8, 1407-1416. [CrossRef] 